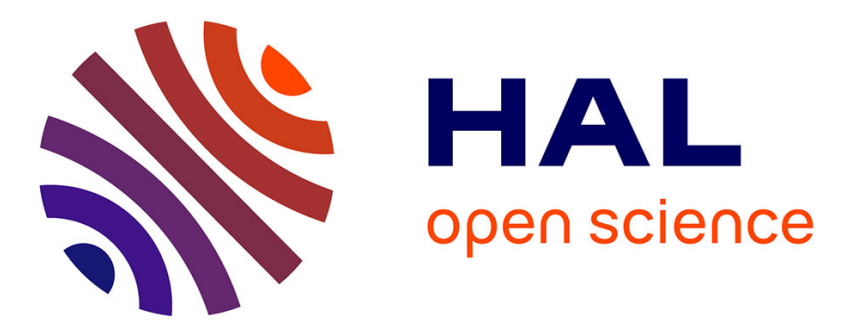

\title{
Flatness-Based Control of an m-Branch Power Flow Controller for Meshed DC Microgrids
}

Xuefang Lin-Shi, Tanguy Simon, Jean-François Trégouët, Hervé Morel

\section{To cite this version:}

Xuefang Lin-Shi, Tanguy Simon, Jean-François Trégouët, Hervé Morel. Flatness-Based Control of an m-Branch Power Flow Controller for Meshed DC Microgrids. PEAS, Nov 2021, Shanghai, China. 10.1109/PEAS53589.2021.9628872 . hal-03375502

\section{HAL Id: hal-03375502 \\ https://hal.science/hal-03375502}

Submitted on 12 Oct 2021

HAL is a multi-disciplinary open access archive for the deposit and dissemination of scientific research documents, whether they are published or not. The documents may come from teaching and research institutions in France or abroad, or from public or private research centers.
L'archive ouverte pluridisciplinaire HAL, est destinée au dépôt et à la diffusion de documents scientifiques de niveau recherche, publiés ou non, émanant des établissements d'enseignement et de recherche français ou étrangers, des laboratoires publics ou privés. 


\section{Flatness-Based Control of an m-Branch Power Flow Controller for Meshed DC Microgrids}

\author{
Xuefang Lin-Shi \\ Univ Lyon, INSA Lyon \\ Université Claude Bernard Lyon 1 \\ Ecole Centrale de Lyon \\ CNRS, Ampère, UMR5005 \\ 69621 Villeurbanne, France \\ xuefang.shi@insa-lyon.fr
}

\author{
Tanguy Simon \\ Univ Lyon, INSA Lyon \\ Université Claude Bernard Lyon 1 \\ Ecole Centrale de Lyon \\ CNRS, Ampère, UMR5005 \\ 69621 Villeurbanne, France \\ tanguy.simon@insa-lyon.fr \\ Hervé Morel \\ Univ Lyon, CNRS, INSA Lyon \\ Université Claude Bernard Lyon 1 \\ Ecole Centrale de Lyon \\ Ampère, UMR5005 \\ 69621 Villeurbanne, France \\ herve.morel@insa-lyon.fr
}

\author{
Jean-François Trégouët \\ Univ Lyon, INSA Lyon \\ Université Claude Bernard Lyon 1 \\ Ecole Centrale de Lyon \\ CNRS, Ampère, UMR5005 \\ 69621 Villeurbanne, France \\ jean-francois.tregouet@insa-lyon.fr
}

\begin{abstract}
The Power flow controller (PFC) is a key element for meshed DC micro-grids. Indeed, this device is able to balance the electric power between the lines of a node in the mesh, which is required to maintain the stability and prevent the overloading of some lines. This paper presents a differential flatness based nonlinear control for this device. The chosen converter topology is modelled by a modular state-space nonlinear system. The primary control objective is to achieve power control on the line connected to each terminal of the PFC despite the uncertainty of the grid. The considered PFC is equipped with a reservoir capacitor. Voltage regulation of this capacitor is also addressed, as a secondary control objective. Simulation results for a 3terminal PFC show the effectiveness of the proposed control. The extension to a 5-terminal PFC demonstrates that the control for an $m$-terminal PFC is easily implemented thanks to the modular structure of the proposed control scheme.
\end{abstract}

Keywords-Meshed DC microgrid, Power flow controller, Power converter, Flatness-based control, Nonlinear control

\section{INTRODUCTION}

Micro-grids are currently being build throughout the world to meet with local energy demands with low carbon emissions [1]. Two of the reasons are their ability to increase the penetration of small renewable energy generators [2] [3] and reduce the transport losses by ensuring that the energy produced is consumed locally. The use of direct current (DC) in microgrids brings various advantages: It removes the problem of reactive power and skin effects. It increases power efficiency by reducing the number of power converters since most generators and loads already have a DC link [4].

Meshed microgrids are another research issue to enhance the reliability, modularity and efficiency of the system while

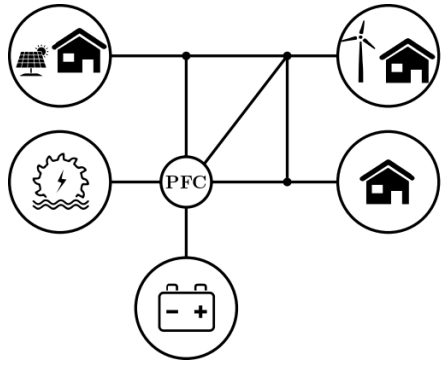

Fig. 1. Micro-grid with a 5-terminal PFC

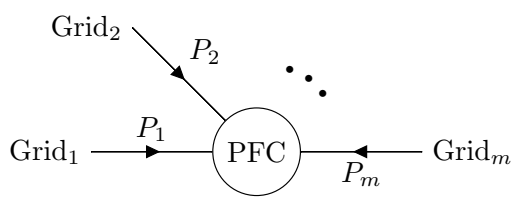

Fig. 2. PFC at an m-terminal node in the grid

reducing the amount of copper used [5]. Moreover, a mesh is the natural structure when loads and generation units are randomly distributed in an area following the argument of Dr. Rik W. De Doncker. In this context, the Power Flow Controller (PFC) must be introduced (Fig. 1). Here, it is a multi-terminal DC/DC converter located at a node of the mesh. It allows to control the power flow in each line of the node (Fig. 2).

The state-of-the-art on the subject can be listed as follows. In [6], the authors propose the use of two split-pi converters where the RST technique with hysteresis switching is used to perform a current-limited voltage controller. The reservoir 


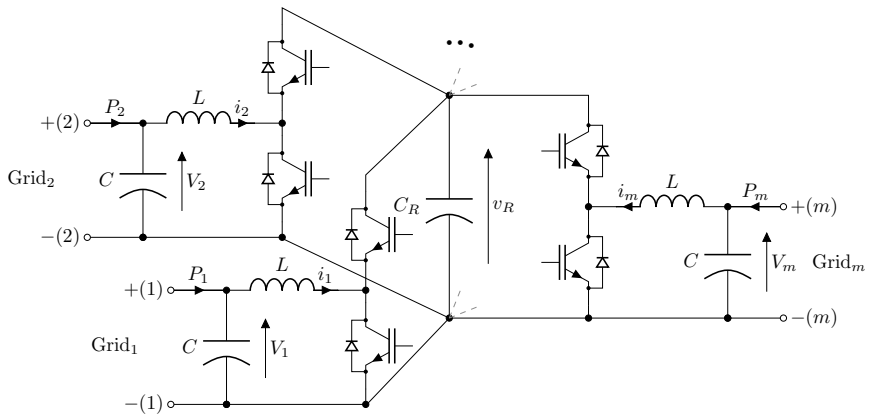

Fig. 3. $m$-terminal topology for PFC proposed in [7]

voltage is properly controlled but power flow control is not achieved. [7] proposed another topology (Fig. 3) consisting of a modified split-pi with $m$ half-bridge legs connected to the same reservoir (or link) capacitor $C_{R}$. This enables power flow control on a multi-terminal node. However, the control proposed in [7] is open-loop and therefore does not guarantee control performances against parameter uncertainties and disturbances. Finally, the reservoir voltage has to be maintained by an additional branch that compensates for the power imbalance. In [8], a new control law is proposed to improve the power control during fluctuations of the reservoir voltage. Unfortunately, the controller regulates the current rather than the power, and the computation of the current reference depends on the voltage at the end of the line. Thus, a fluctuation in the loads or generation units of the grid would lead to a steady-state error of the power. Furthermore, the reservoir voltage remains uncontrolled and therefore the physical boundaries cannot be guaranteed. Moreover, in the three latter articles, no dynamic model of the system is given, and no mathematical guarantee for stability is found.

In the authors' previous work [9], the topology of Fig. 3 with a 3-terminal PFC is investigated. It is first modelled as a nonlinear multivariable system. Then, this state model is augmented with integrators on the tracking errors. This augmented model is linearised and a multivariable ProportionalIntegral control law is derived by pole placement. It achieves simultaneous direct balancing control of the power in each line and of the voltage across the reservoir capacitor. The main drawbacks of this latter technique is the dependency of the control gain matrices to parameter variations, resulting in a limited robustness, as well as the linearisation process which only ensures local stability in the state-space. To the knowledge of the authors, no feedback control law for a PFC with more than 3 terminals has been published in the literature.

In this paper, the $m$-terminal PFC topology shown in Fig. 3 is studied. It is made of $m$ identical buck-boost converters connected in parallel to a unique reservoir capacitor noted $C_{R}$. Each branch (buck-boost) is connected to a part of the grid as shown in Fig. 1 and 2. The objective of the PFC is to control the electric power in the lines it is connected to. This can be performed only if $v_{R}$ is controlled. To achieve simultaneous direct balancing control of the power in each line and the control of the capacitor voltage, a nonlinear two-level control scheme based on the differential flatness theory [10] is proposed. This principle has been applied to many power electronics systems [11]. In the present case, the dynamics of each control loop are defined by a trajectory planning where each power control loop can be considered as "decentralized" and is easy to be duplicated. This facilitates the control design and its implementation. Thanks to the modularity of the proposed control law, the extension to an $m$-terminal PFC becomes trivial.

The paper is organized as follows. In the second section, the dynamical equations of an $m$-terminal PFC are provided. The third section is devoted to the design of the differential flatness-based control laws. The flatness differential theory is first recalled. Then, the flat outputs are chosen to demonstrate the flat property of the line power part and capacitor energy part. A flatness-based control law is designed for each part. The fourth section focuses on simulation results on a threeterminal PFC and on an extended five-terminal one.

\section{PFC MODELLING}

To derive the control law, a simplified averaged model over a switching period is adopted for the chosen PFC topology of Fig. 3, by taking the following assumption:

Assumption 1. The line voltage $V_{k}$ is considered as a strictly positive measurable constant, and the reservoir voltage $v_{R}$ is always strictly positive.

This assumption is justified knowing that, in practice, the line voltage of a grid always stays in a neighbourhood of the nominal voltage, a positive value well above zero. Moreover, as can be seen on Fig. 3, if $v_{R}<V_{k}$, the anti-parallel diode of each high-side IGBT opens, charging the reservoir capacitor until $v_{R} \approx \max _{k}\left(V_{k}\right)>0$.

The components are assumed to be ideal. By neglected converter losses and grid element dynamics, an $m$-terminal node PFC can be modelled as:

$$
\begin{aligned}
P_{k} & =V_{k} i_{k} \\
\frac{d i_{k}}{d t} & =\frac{1}{L}\left(V_{k}-v_{R} d_{k}\right) \\
\frac{d v_{R}}{d t} & =\frac{1}{C_{R}} \sum_{k=1}^{m} i_{k} d_{k}=\frac{1}{C_{R}} \sum_{k=1}^{m} \frac{P_{k}}{V_{k}} d_{k}
\end{aligned}
$$

where $P_{k}, V_{k}$ and $i_{k}$ are respectively the averaged power, voltage and current of the $k$-th branch, $d_{k}$ is the corresponding pulse width modulation (PWM) duty ratio, $v_{R}$ is the averaged reservoir voltage across the reservoir capacitor $C_{R}$.

By assuming that the reservoir capacitor dynamic is slower than that of the currents in each line, the dynamical system (1) can be seen as a two-time scale system. A mathematical formalism has been developed for two time-scale systems (corresponding to the so-called "frequency separation" in the linear system framework). It is called Singular Perturbations and is well explained in [12, Ch.11]. The idea is that if both the fast and slow subsystems are exponentially stable under 


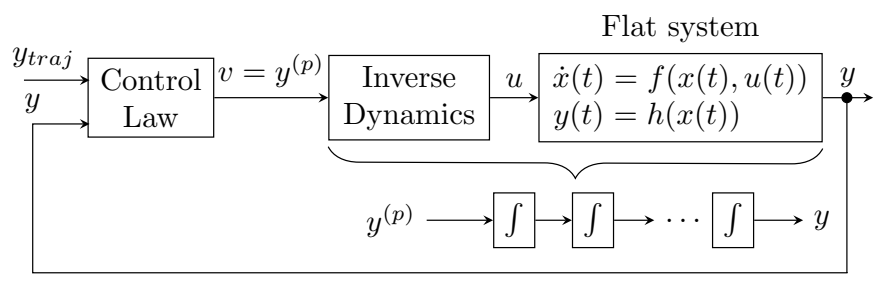

Fig. 4. Concept of flatness-based control

time-scale separation assumptions, then the global system is also exponentially stable for sufficient time-scale separation. The fast time-scale subsystem is composed of the current of each branch $i_{k}$, and the slow time-scale system is composed of the reservoir voltage $v_{R}$.

The control objective is to track the power reference for every branch $k$ and to control the reservoir voltage to ensure the proper operation of the PFC [9]. The strategy proposed in this paper is to use a differential flatness-based control law [13] for the both fast and slow subsystems to track respectively the power reference $P_{k}^{r}$ for all $k \in\{1, \ldots, m\}$ and the capacitor voltage $v_{R}$. Among the $k$ power references, $k-1$ are given by a higher-layer control algorithm while the last one is defined by the reservoir voltage controller, as shown in Fig. 5.

\section{FLATNESS-BASED CONTROL DESIGN}

\section{A. Brief Recall of Differential Flatness Theory}

The concept of flat systems was introduced by Fliess et al. using the formalism of differential algebra [10]. Consider a nonlinear system of the form:

$$
\dot{x}(t)=f(x(t), u(t)),
$$

where $x(t) \in \mathbb{R}^{n}, u(t) \in \mathbb{R}^{m}$ and $m \leq n$. This system is considered differentially flat if there exists a flat output. An output $y$ is flat if there exists the following smooth functions [14, Ch2]

$$
\begin{aligned}
& h: \mathbb{R}^{n} \times\left(\mathbb{R}^{m}\right)^{p+1} \rightarrow \mathbb{R}^{m} \\
& \eta:\left(\mathbb{R}^{m}\right)^{p} \rightarrow \mathbb{R}^{n} \\
& \vartheta:\left(\mathbb{R}^{m}\right)^{p+1} \rightarrow \mathbb{R}^{m}
\end{aligned}
$$

such that

$$
\begin{aligned}
& y=h\left(x, u, \dot{u}, \ldots, u^{(p)}\right) \\
& x=\eta\left(y, \dot{y}, \ldots, y^{(p-1)}\right) \\
& u=\vartheta\left(y, \dot{y}, \ldots, y^{(p)}\right)
\end{aligned}
$$

where $p$ can be arbitrarily large [14, p82]. This means that all the state vector $x$ of the differentially flat system and its control inputs $u$ can be expressed as a function of the flat output and flat output's derivatives.

The flat system dynamic behaviour is determined by its output. If the output vector $y$ can be demonstrated to be flat, the design of a control law capable of tracking every desired trajectory of the flat output $y_{t r a j}$ becomes straightforward, see Fig. 4 and [13]). It is easy to demonstrate that the following feedback control law ensures that the tracking errors $e=\left(y-y_{\text {traj }}\right)$ asymptotically vanish $[14, \mathrm{p} 81]$ :

$$
\begin{aligned}
y^{(p)}-y_{\text {traj }}^{(p)} & +K_{p-1}\left(y^{(p-1)}-y_{\text {traj }}^{(p-1)}\right) \\
& +\cdots+K_{0}\left(y-y_{\text {traj }}\right)=0,
\end{aligned}
$$

where the set of controller parameters $K_{p-1}, \ldots, K_{0}$ are chosen such that the closed-loop characteristic polynomial is Hurwitz. Unlike input-output linearization control for nonlinear dynamical systems, differential flatness-based control does not lead to zero dynamics [15, Ch.4]. As a consequence, the stability of the whole closed-loop system of Fig. 4 is guaranteed.

Trajectory planning is an important step in the implementation of a flatness-based control. Since all the state and input variables are to be written as functions of a chosen flat output $y$, the flat output trajectory $y_{\text {traj }}$ defines the trajectories of all the state or input variables. Therefore, a well-known waveform can be considered such that all the transient state behaviors can be predicted.

\section{B. Design of the Power Control Loop}

The power control applies for the fast time-scale subsystem (1b) with the state vector $x:=\left[i_{1}, i_{2}, \ldots, i_{m}\right]^{\top}$, and the control vector $u:=\left[d_{1}, d_{2}, \ldots, d_{m}\right]^{\top}$. Before designing this control loop for the fast subsystem, the following assumption has to be considered:

Assumption 2 (time-scale separation I). The dynamic of the slow subsystem (reservoir capacitor voltage $v_{R}$ ) is infinitely slow compared to the fast one, i.e. it can be neglected for the fast subsystem (current of each line $i_{k}$ ) control loop design.

Note that this assumption is only taken for the fast control loop design. The flat output candidate is chosen as the power of each branch noted $y:=\left[P_{1}, P_{2}, \ldots, P_{m}\right]^{\top}$. Indeed, (3) is verified since for all $k \in\{1, \ldots, m\}$ :

$$
\begin{aligned}
y_{k} & =x_{k} V_{k}=h\left(x_{k}\right), \\
x_{k} & =\frac{y_{k}}{V_{k}}=\eta\left(y_{k}\right), \\
u_{k} & =\left(V_{k}-\frac{L \dot{y}_{k}}{V_{k}}\right) \frac{1}{v_{R}}=\vartheta\left(\dot{y}_{k}\right) .
\end{aligned}
$$

Note that in under Assumptions $1 \& 2, V_{k}$ and $v_{R}$ are strictly positive, constant and measurable.

Thus, subsystem (1b) can be considered as a flat system with $y=\left[P_{1}, P_{2}, \ldots, P_{m}\right]^{\top}$ as the flat output.

Then, to track the $k$-th power trajectory $P_{k, t r a j}$ for all $k \in$ $\{1, \ldots, m\}$, a feedback control law is given as follows:

$$
\begin{aligned}
\dot{P}_{k}=\dot{P}_{k, t r a j} & -K_{p k}\left(P_{k}-P_{k, t r a j}\right) \\
& -K_{i k} \int_{0}^{t}\left(P_{k}(\tau)-P_{k, t r a j}(\tau)\right) d \tau
\end{aligned}
$$

where the integral action is added to eliminate the disturbances and to compensate for model errors. The control parameters $K_{p k}$ and $K_{i k}$ are chosen to ensure the appropriate $k$-th power 


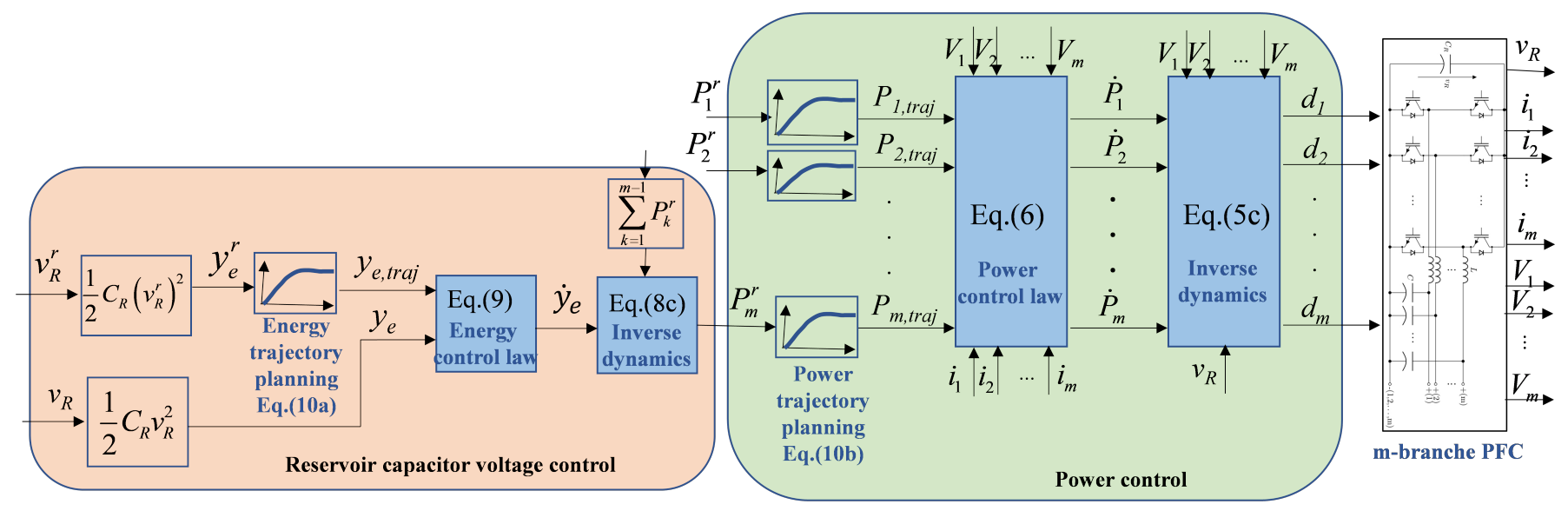

Fig. 5. Differential flatness-based control scheme for an m-branch PFC

reference tracking dynamics, i.e. the dynamics of the tracking errors $e_{k}=\left(P_{k}-P_{k, t r a j}\right)$.

\section{Design of the Reservoir Capacitor Voltage Control Loop}

The capacitor voltage dynamics corresponds to the slow time-scale subsystem (1c). For the sake of simplicity, only the power in the $m$-th branch is adjusted to regulate the reservoir voltage: while $P_{1}^{r}$ to $P_{m-1}^{r}$ are defined by the microgrid supervising algorithm, $P_{m}^{r}$ is defined by the reservoir voltage control loop. (see Fig. 5).

Assumption 3 (time-scale separation II). The regulation of the power in each branch is fast enough to consider that $P_{k}=P_{k}^{r}$ for all $k \in\{1, \ldots, m\}$. The current dynamics can therefore be neglected.

The previous assumption is only taken for the design of the slow-scale time control loop (section III-C). This assumption is also known as the quasi steady state of the fast subsystem. Under this assumption, (1b) gives $V_{k}=v_{R} d_{k}$. (1c) can thus be written as:

$$
\begin{aligned}
\frac{d v_{R}}{d t} & =\frac{1}{C_{R} v_{R}}\left(\sum_{k=1}^{m-1} P_{k}^{r}+P_{m}^{r}\right) \\
& =f\left(x_{e}\right)+g\left(x_{e}\right) u_{e},
\end{aligned}
$$

where the new state variable is defined as $x_{e}=v_{R}$ and the new control input as $u_{e}=P_{m}^{r}$. The proposed flat output candidate is the energy of the capacitor $y_{e}=\frac{1}{2} C_{R} v_{R}^{2}$. Since

$$
\dot{y}_{e}=C_{R} v_{R} \frac{d v_{R}}{d t}=\sum_{k=1}^{m-1} P_{k}^{r}+P_{m}^{r}=\sum_{k=1}^{m-1} P_{k}^{r}+u_{e},
$$

and under Assumption 3, (3) is verified:

$$
\begin{aligned}
& y_{e}=\frac{1}{2} C_{R} x_{e}^{2}, \\
& x_{e}=\sqrt{\frac{2 y_{e}}{C_{R}}}, \\
& u_{e}=P_{m}^{r}=\dot{y}_{e}-\sum_{k=1}^{m-1} P_{k}^{r} .
\end{aligned}
$$

Thus, the differential flatness conditions expressed in (3) are verified, and system (1c) is a flat system with $y_{e}=\frac{1}{2} C_{R} v_{R}^{2}$ as the flat output and $u_{e}$ defined in (8c) as the flatness-based control (inverse dynamics) which will be applied to generate the last power reference for the fast time-scale subsystem.

As for the power control, to track the desired energy trajectory $y_{e, t r a j}$ which can be generated from the desired capacitor voltage $v_{R}^{r}$, the feedback control law is given as follows:

$$
\begin{aligned}
\dot{y}_{e}=\dot{y}_{e, r e f} & -K_{p e}\left(y_{e}-y_{e, t r a j}\right) \\
& -K_{i e} \int_{0}^{t}\left(y_{e}(\tau)-y_{e, t r a j}(\tau)\right) d \tau,
\end{aligned}
$$

where $K_{p e}$ and $K_{i e}$ define the dynamics of the reservoir energy tracking error $\left(y_{e}-y_{e, t r a j}\right)$. They are chosen to ensure the stability and robustness of the energy tracking which drives the capacitor voltage $v_{R}$ to its reference $v_{R}^{r}$.

\section{Trajectory Planning and Global Control Scheme}

As underlined in subsection III.A, the flat output trajectory defines the trajectories of all the state or input variables. Physical constrains and time-scale separation assumptions can be taken into account in the trajectory planning. To limit the derivative terms in the control laws (6) and (9), two lowpass second-order filters are used to generate the desired trajectories respectively for the flat outputs $y_{e, t r a j}$ (energy) and $P_{k, t r a j}$ (power) components. The transfer functions are given respectively by

$$
\begin{aligned}
\frac{y_{e, t r a j}(s)}{y_{e}^{r}(s)} & =\frac{\omega_{t e}^{2}}{s^{2}+2 \xi_{t e} \omega_{t e} s+\omega_{t e}^{2}} \\
\frac{P_{k, t r a j}(s)}{P_{k}^{r}(s)} & =\frac{\omega_{t k}^{2}}{s^{2}+2 \xi_{t k} \omega_{t k} s+\omega_{t k}^{2}}
\end{aligned}
$$

where $\xi_{t e}$ (respectively $\xi_{t k}$ ) and $\omega_{t e}$ (respectively $\omega_{t k}$ ) are the desired dominant damping ratio and natural frequency, respectively. These parameters fix the dynamics of the trajectories to be tracked, and the two time-scale properties (time-scale separation assumptions) are ensured by choosing $\omega_{t e}<<\omega_{t k}$. 


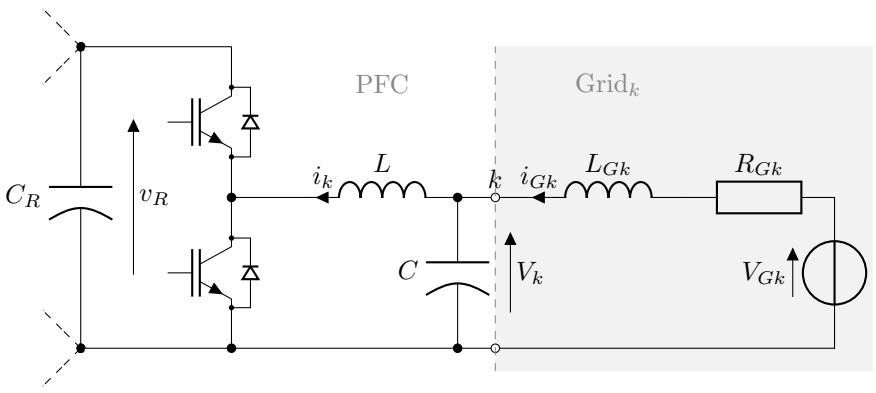

Fig. 6. $k$-th branch of the PFC (left) and the proposed grid model as seen by this terminal (right)

Fig. 5 shows the functional diagram summarising the global control algorithm. Note that the measured currents $i_{k}$ are only used in (6) to determine $P_{k}$ for power trajectory tracking, but not in the inverse dynamic which may be sensitive to measured noises.

\section{SIMULATION RESULTS}

To validate the proposed control law, simulations have been made through Matlab/Simulink. The averaged model chosen for the simulation is the one given by the equation (2) of [9], where each terminal is cabled as in Fig. 6 by added to (1) the dynamics of $V_{k}$ and the grid model. This grid is modelled as a constant voltage source $V_{G k}$ and a line modelled by an inductance $L_{G k}$ with a resistance $R_{G k}$. Different $L_{G k}, R_{G k}$ and $V_{G k}$ can be considered for each terminal. Each branch of the PFC (Fig. 6) has identical parameters $L=0.75 \mathrm{mH}$, $C=20 \mu \mathrm{F}$ with reservoir capacitor $C_{R}=60 \mu \mathrm{F}$.

As explained in III.D, $\xi_{t e}$ and $\omega_{t e}$ fix the dynamic behavior of the energy trajectory (i.e. capacitor voltage dynamics). $\xi_{t k}$ and $\omega_{t k}$ define the dynamics of the power trajectories. $\xi_{t e}$ and $\xi_{t k}$ are taken equal to $1 . \omega_{t e}=100 \mathrm{rad} . \mathrm{s}^{-1}$ and $\omega_{t k}=$ $2000 \mathrm{rad}_{\mathrm{s}}^{-1}$ are chosen to ensure the time-scale separation assumptions $2 \& 3$. The control parameters for error tracking of (6) and (9) are chosen as: $K_{p k}=2 \xi_{p} \omega_{p}, K_{i k}=\omega_{p}^{2}, K_{p e}=$ $2 \xi_{e} \omega_{e}$ and $K_{i e}=\omega_{e}^{2}$, where $\xi_{p}=\xi_{e}=0.7, \omega_{p}=1000$ rad.s ${ }^{-1}$ and $\omega_{e}=100$ rad.s ${ }^{-1}$. These control parameters ensure an asymptotic convergence of the power and energy errors to zero.

\section{A. 3-terminal simulation}

Firstly, the control of a 3-terminal PFC is simulated. The line parameters are $L_{G 1,2,3}=\left[\begin{array}{lll}18 & 18 & 18\end{array}\right] \mu \mathrm{H}, R_{G 1,2,3}=$ $\left[\begin{array}{lll}2.6 & 30.3 & 1.4\end{array}\right] \Omega, V_{G 1,2,3}=\left[\begin{array}{lll}400 & 383 & 402\end{array}\right] \mathrm{V}$.

At $t=0.04 \mathrm{~s}$, the references are changed from $\left[P_{1}^{r}, P_{2}^{r}, v_{R}^{r}\right]$ $=[-600 \mathrm{~W},-200 \mathrm{~W}, 500 \mathrm{~V}]$ to $[-900 \mathrm{~W}, 100 \mathrm{~W}, 500 \mathrm{~V}]$. To assess the robustness of the proposed control law against the grid uncertainty, the voltage $V_{G 1}$ at the end of the line 1 is changed form $400 \mathrm{~V}$ to $300 \mathrm{~V}$ at $t=0.06 \mathrm{~s}$.

The simulation results are given in Fig. 7. It can be seen that the capacitor voltage $v_{R}$ and the powers $P_{1}, P_{2}, P_{3}$ converge from the initial condition to their reference (blue curves) without static error. The transition performance of the power tracking can be appreciated, notably at $t=0.04 \mathrm{~s}$ when
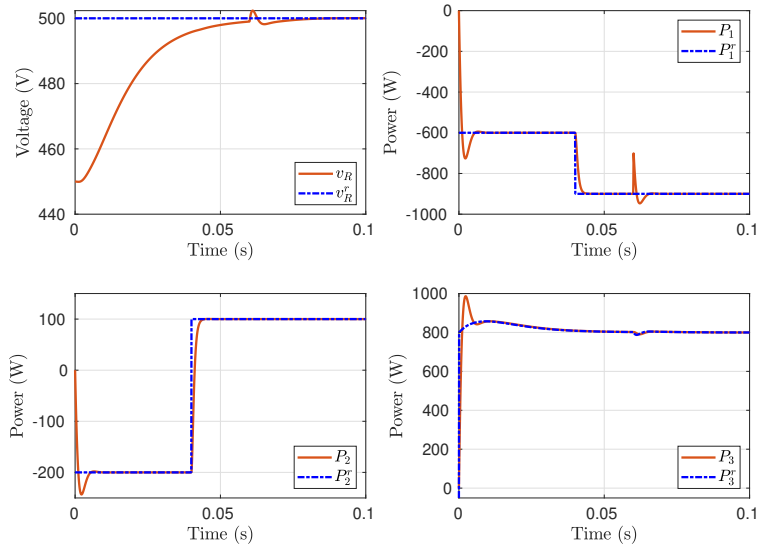

Fig. 7. Simulation results for a three -terminal PFC : reservoir voltage and power in each line with their references

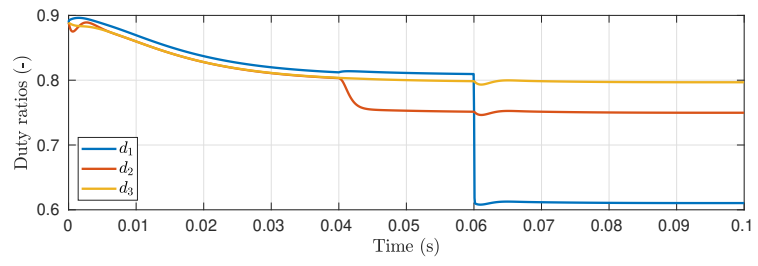

Fig. 8. Simulation results for a three -terminal PFC : duty cycles

the power references change. At $t=0.06 \mathrm{~s}$, the grid variation induces a little oscillation of $v_{R}$ and $P_{1}$, but return quickly to their reference. This demonstrates some robustness of the proposed control, especially since the perturbation is very large $(100 \mathrm{~V})$. Fig. 8 displays the control signal (duty ratio) of each branch. It can be verified that they are do not saturate.

\section{B. 5-terminal simulation}

Secondly, the simulation is extended to a five-terminal PFC. The converter parameters are unchanged, with $L=$ $0.75 \mathrm{mH}, C=20 \mu \mathrm{F}$ and $C_{R}=60 \mu \mathrm{F}$. The line parameters are $L_{G 1,2,3,4,5}=\left[\begin{array}{lllll}18 & 18 & 18 & 18 & 18\end{array}\right] \mu \mathrm{H}$, $R_{G 1,2,3,4,5}=\left[\begin{array}{lllll}2.6 & 30.3 & 2.6 & 30.3 & 1.4\end{array}\right] \Omega, V_{G 1,2,3,4,5}=$ $\left[\begin{array}{lllll}400 & 383 & 400 & 383 & 402\end{array}\right]$ V. The same set of control parameters is kept. As for the 3-terminal PFC, the references are changed from $\left[P_{1}^{r}, P_{2}^{r}, P_{3}^{r}, P_{4}^{r}, v_{R}^{r}\right]$ $=[-600 \mathrm{~W},-200 \mathrm{~W},-600 \mathrm{~W},-200 \mathrm{~W}, 500 \mathrm{~V}]$ to $[-900 \mathrm{~W}, 100 \mathrm{~W},-200 \mathrm{~W},-600 \mathrm{~W}, 500 \mathrm{~V}]$ at $t=0.04 \mathrm{~s}$. To assess the robustness of the proposed control law against the grid uncertainty, the voltage $V_{G 1}$ at the end of the line 1 is changed form $400 \mathrm{~V}$ to $300 \mathrm{~V}$ at $t=0.06 \mathrm{~s}$.

The simulation results are given in Fig. 9. As for the 3terminal PFC, same transition and steady-state performances can be observed for 5-terminal PFC since the same trajectory planning and control parameters are applied. Note that the steady-state value of the last power $P_{5}$ corresponds to the balanced power of all the other line powers. For the same grid variation at $t=0.06 \mathrm{~s}$, the overshoot of $v_{R}$ is about $506 \mathrm{~V}$ 

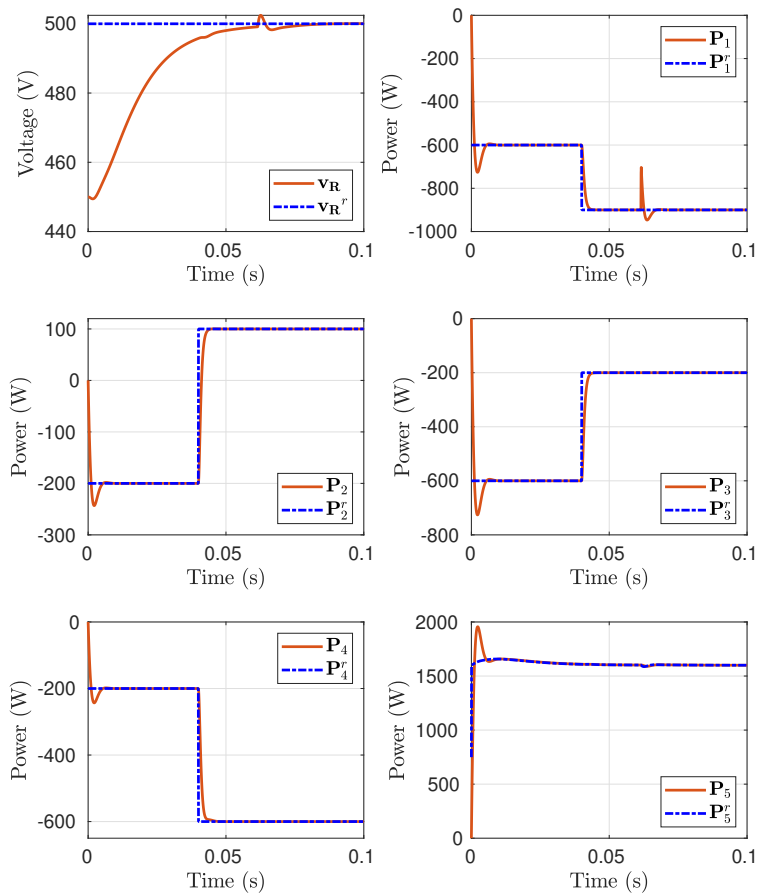

Fig. 9. Simulation results for a five-terminal PFC : reservoir voltage and power in each line with their references.

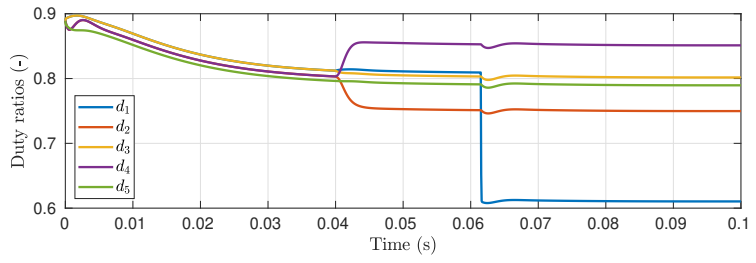

Fig. 10. Simulation results for a five-terminal PFC : duty cycles

instead of $502 \mathrm{~V}$ for 3-branch. The control signals, pictured in Fig. 10, are all unsaturated $\left(d_{k} \in[0,1]\right)$. The big change of $d_{1}$ can be observed at $t=0.06 \mathrm{~s}$ due to the grid perturbation on line 1 .

\section{Conclusions}

In this paper, a differential flatness-based control law has been proposed both for line power control and for the converter capacitor reservoir energy (i.e. voltage) control in a PFC for mesh DC microgrids. The proposed control scheme has been successfully validated in the case of a three-terminal PFC and a five-terminal PFC. This last case shows that it is easy to extended the control scheme to any $m$-terminal PFC. Despite the demanding assumptions on the modelling and on the timescale separation, the transition and steady state performances as well as the robustness of the control law against grid disturbances are asserted through simulations. Few control parameters are required (two for capacitor energy control, two for each terminal power control) and are easily tuned. Experimental validation of the theoretical results will be made in a laboratory test bench for future work.

\section{REFERENCES}

[1] (2021) Microgrid projects. [Online]. Available: http://microgridprojects.com/

[2] S. Sen and V. Kumar, "Microgrid control: A comprehensive survey," Annual Reviews in control, vol. 45, pp. 118-151, 2018.

[3] D. Kumar, F. Zare, and A. Ghosh, "DC Microgrid Technology: System Architectures, AC Grid Interfaces, Grounding Schemes, Power Quality, Communication Networks, Applications, and Standardizations Aspects," IEEE Access, vol. 5, pp. 12230-12 256, 2017. [Online]. Available: http://ieeexplore.ieee.org/document/7937799/

[4] A. T. Elsayed, A. A. Mohamed, and O. A. Mohammed, "DC microgrids and distribution systems: An overview," Electric Power Systems Research, vol. 119, pp. 407-417, Feb. 2015. [Online]. Available: https://linkinghub.elsevier.com/retrieve/pii/S0378779614003885

[5] L. Mackay, "Toward the Universal DC Distribution System," pp. 1032 1042, Jul. 2017.

[6] M. Barara, H. Morel, and G. Clerc, "Control Strategy Scheme for Consistent Power Flow Control in Meshed DC Micro-grids," 2017, pp. $1-10$.

[7] Y. Takahashi, K. Natori, and Y. Sato, "A multi-terminal power flow control method for next-generation DC power network," in 2015 IEEE Energy Conversion Congress and Exposition (ECCE). Montreal, QC, Canada: IEEE, Sep. 2015, pp. 6223-6230. [Online]. Available: http://ieeexplore.ieee.org/document/7310533/

[8] K. Natori, Y. Nakao, and Y. Sato, "A Novel Control Approach to Multi-Terminal Power Flow Controller for Next-Generation DC Power Network," in 2018 International Power Electronics Conference (IPECNiigata 2018 -ECCE Asia), May 2018, pp. 588-592.

[9] T. Simon, J.-F. Trégouët, X. Lin-Shi, and H. Morel, "Modelling and Control of a Power Flow Controller for DC Microgrids." Ghent, Belgium: IEEE, 2021.

[10] M. Fliess, J. Lévine, P. Martin, and P. Rouchon, "Flatness and defect of non-linear systems: introductory theory and examples," International Journal of Control, vol. 61, no. 6, pp. 1327-1361, Jun. 1995. [Online]. Available: https://www.tandfonline.com/doi/full/10.1080/00207179508921959

[11] M. Soheil-Hamedani, M. Zandi, R. Gavagsaz-Ghoachani, B. NahidMobarakeh, and S. Pierfederici, "Flatness-based control method: A review of its applications to power systems," in 2016 7th Power Electronics and Drive Systems Technologies Conference (PEDSTC). IEEE, 2016, pp. $547-552$.

[12] H. K. Khalil, "Nonlinear systems," 2002.

[13] P. Thounthong, S. Sikkabut, N. Poonnoy, P. Mungporn, B. Yodwong, P. Kumam, N. Bizon, B. Nahid-Mobarakeh, and S. Pierfederici, "Nonlinear Differential Flatness-Based Speed/Torque Control With StateObservers of Permanent Magnet Synchronous Motor Drives," IEEE Transactions on Industry Applications, vol. 54, no. 3, pp. 2874-2884, May 2018, conference Name: IEEE Transactions on Industry Applications.

[14] G. G. Rigatos, Nonlinear control and filtering using differential flatness approaches: applications to electromechanical systems. Springer, 2015, vol. 25.

[15] A. Isidori, E. Sontag, and M. Thoma, Nonlinear control systems. Springer, 1995, vol. 3. 